\title{
Development of NIR detectors and science-driven requirements for SNAP
}

M. G. Brown, C. Bebek, G. Bernstein, A. Bonissent, B. Carithers, et al.

M. G. Brown, C. Bebek, G. Bernstein, A. Bonissent, B. Carithers, D. Cole, D. Figer, D. Gerdes, L. Gladney, W. Lorenzon, A. Kim, G. Kushner, N. Kuznetsova, M. Lampton, M. Levi, E. Linder, S. McKee, R. Miquel, N. Mostek, S. Mufson, S. Perlmutter, M. Schubnell, S. Seshadri, H. Shukla, R. Smith, A. Stebbins, C. Stoughton, G. Tarlé, "Development of NIR detectors and science-driven requirements for SNAP," Proc. SPIE 6265, Space Telescopes and Instrumentation I: Optical, Infrared, and Millimeter, 626535 (5 July 2006); doi: 10.1117/12.672141

Event: SPIE Astronomical Telescopes + Instrumentation, 2006, Orlando, Florida, United States 


\title{
Development of NIR Detectors and Science Driven Requirements for SNAP
}

\author{
M. G. Brown ${ }^{a}$, C. Bebek ${ }^{b}$, G. Bernstein ${ }^{c}$, A. Bonissent $^{d}$, B. Carithers ${ }^{b}$, D. Cole $^{e}$, D. Figer $^{f}$, \\ D. Gerdes ${ }^{a}$, L. Gladney ${ }^{c}$, W. Lorenzon ${ }^{a}$, A. Kim $^{b}$, G. Kushner ${ }^{b}$, N. Kuznetsova ${ }^{b}$,

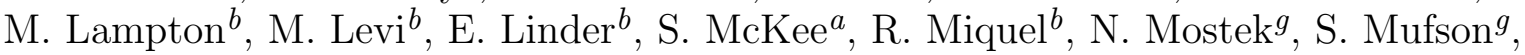 \\ S. Perlmutter ${ }^{b}$, M. Schubnell ${ }^{a}$, S. Seshadrie ${ }^{2}$ H. Shukla ${ }^{b}$, R. Smith ${ }^{h}$, A. Stebbins ${ }^{i}$, \\ C. Stoughton ${ }^{i}$, and G. Tarlé ${ }^{a}$ for the SNAP Collaboration \\ ${ }^{a}$ University of Michigan, Ann Arbor MI, USA \\ ${ }^{b}$ Lawrence Berkeley National Laboratory, Berkeley CA, USA \\ ${ }^{c}$ University of Pennsylvania, Philadelphia PA, USA \\ ${ }^{d}$ CNRS/IN2P3/CPPM, Marseille, France \\ ${ }^{e}$ Jet Propulsion Lab, Pasadena CA, USA \\ ${ }^{f}$ Space Telescope Science Institute, Baltimore MD, USA \\ Indiana University, Bloomington IN, USA \\ ${ }^{h}$ California Institute of Technology, Pasadena CA, USA \\ ${ }^{i}$ Fermi National Accelerator Laboratory, Batavia IL, USA
}

\begin{abstract}
Precision near infrared (NIR) measurements are essential for the next generation of ground and space based instruments. The SuperNova Acceleration Probe (SNAP) will measure thousands of type Ia supernovae up to a redshift of 1.7. The highest redshift supernovae provide the most leverage for determining cosmological parameters, in particular the dark energy equation of state and its possible time evolution. Accurate NIR observations are needed to utilize the full potential of the highest redshift supernovae. Technological improvements in NIR detector fabrication have lead to high quantum efficiency, low noise detectors using a $\mathrm{HgCdTe}$ diode with a band-gap that is tuned to cutoff at $1.7 \mu \mathrm{m}$. The effects of detector quantum efficiency, read noise, and dark current on lightcurve signal to noise, lightcurve parameter errors, and distance modulus fits are simulated in the SNAPsim framework. Results show that improving quantum efficiency leads to the largest gains in photometric accuracy for type Ia supernovae. High quantum efficiency in the NIR reduces statistical errors and helps control systematic uncertainties at the levels necessary to achieve the primary SNAP science goals.
\end{abstract}

Keywords: NIR detectors, HgCdTe, supernova, dark energy, SNAP

\section{INTRODUCTION}

Recent observations show that the expansion of the universe is accelerating. ${ }^{1,2}$ The energy content responsible for the acceleration is called dark energy, and little is known about its nature. One model for dark energy is the cosmological constant, initially proposed by Einstein to balance the attractive matter and result in a static universe. This is equivalent to a vacuum energy, with constant density throughout space and time. Dark energy affects the expansion history of the universe, which can be measured with standard candles like type Ia supernovae. Measuring the properties of dark energy and understanding its nature is one of the most challenging problems in physics today.

The field of cosmology is evolving into a precision science, with a small number of parameters describing the observable universe. The current generation of experiments can measure most of the parameters to an accuracy of about $10 \%$. The next generation of instruments will push these constraints down to the $2 \%$ level, where control

E-mail: brownmg@umich.edu

D. Figer is now at Rochester Institute of Technology, Rochester NY, USA

Space Telescopes and Instrumentation I: Optical, Infrared, and Millimeter, edited by John C. Mather, Howard A. MacEwen, Mattheus W.M. de Graauw,

Proc. of SPIE Vol. 6265, 626535, (2006) · 0277-786X/06/\$15 · doi: 10.1117/12.672141

Proc. of SPIE Vol. 6265 626535-1 
of systematic errors drives the experimental procedure. For type Ia supernovae measurements the parameters of interest are: $H_{0}, \Omega_{M}, \Omega_{X}, w_{0}$, and $w^{\prime} . H_{0}$ is the Hubble constant; it sets the overall distance scale and age of the universe. The matter and dark energy density, $\Omega_{M}$ and $\Omega_{X}$, describe the total energy content of the universe. The density is defined in critical units, $\Omega \equiv \rho / \rho_{c}$, where $\rho_{c}$ is the density required to have a spatially flat geometry. The final two parameters describe the dark energy equation of state, defined as the ratio of the pressure to the energy density, $w(z) \equiv p(z) / \rho(z)=w_{0}+w^{\prime} z$, with $w^{\prime} \equiv d w / d z(z=0)$. The equation of state determines how the energy density scales with expansion: $\rho=\rho_{0} a(t)^{-3(1+w)}$ for a constant equation of state, $w$. $a(t)$ is the cosmic scale factor, which sets the relative length scale in the universe over time. The redshift is defined by the total expansion from time $t_{0}$ to $t, 1+z \equiv a\left(t_{0}\right) / a(t)$, and by convention $a\left(t_{0}\right)=1$. Ordinary matter is pressure-less, with $w=0$ and $\rho \propto a(t)^{-3}$; a cosmological constant, $w=-1$, has a constant energy density over time. Measurements from the cosmic microwave background, large scale structure, and type Ia supernovae have converged on a 'concordance' cosmology with $H_{0}=70 \mathrm{~km} \mathrm{~s}^{-1} \mathrm{Mpc}^{-1}, \Omega_{M}=0.3, \Omega_{\Lambda}=0.7$, and $w=-1$. The total energy density is one, indicating a flat Euclidean geometry, consistent with predictions of inflation. Replacing $\Omega_{X}$ with $\Omega_{\Lambda}$ indicates that the dark energy is assumed to be a cosmological constant. This model is known as the flat $\Lambda \mathrm{CDM}$ (cold dark matter) cosmology.

Missions are now being designed to explore dark energy. The SuperNova Acceleration Probe (SNAP) ${ }^{3}$ is one such mission that will measure dark energy using a variety of complementary techniques, including weak lensing, baryon acoustic oscillations, and type Ia supernovae as standard candles. Of these techniques, type Ia supernovae are currently the most well studied and understood method. Systematic uncertainties are controlled by observing every supernova through 9 different filters and obtaining a spectrum near peak brightness. There is long-standing evidence that the spectral features and colors allow the supernovae to be divided into homogeneous subclasses, ${ }^{4}$ and advances in supernova theory are helping to explain the differences in peak brightness based on the progenitor properties and environment. The other methods provide additional constraints and can break degeneracies between e.g. the dark energy equation of state and the matter density. The systematic uncertainties in weak lensing and baryon oscillation measurements are not yet completely understood, but both methods have the potential to constrain dark energy with similar precision to type Ia supernovae.

Type Ia supernovae are thermonuclear explosions of carbon-oxygen white dwarfs at the Chandrasekhar mass, $1.4 M_{\odot}$. The events are extremely bright, often brighter than their host galaxies, and correlations between the peak brightness and the lightcurve decline rate reduce the dispersion in peak brightness to only $0.12-0.15$ mag in the restframe B and V bands. Observations of supernovae over a range of redshifts map the expansion history of the universe according to the magnitude-redshift relationship. Different dark energy models and energy densities lead to different expansion histories. In the nearby universe it is difficult to discriminate between the models, but at high redshift the models diverge, providing information about the nature of dark energy. The restframe optical light from high redshift supernovae is observed in the NIR. These are the most difficult and time consuming (spectroscopic exposure times scale as $(1+z)^{6}$ ) events to observe, but they provide the most leverage on the dark energy equation of state. The simulations presented focus on the distance modulus, $\mu$, error for type Ia supernovae, where $\mu \equiv m-M$, the apparent minus absolute magnitude. Cosmology fitting to extract the errors on dark energy parameters is straightforward, but is not addressed due to space constraints. The focus of this work is to explore the NIR detector properties needed to reduce the statistical errors to the systematic uncertainty limit. NIR observations of the restframe B and V band lightcurves for high redshift type Ia supernovae help control the systematic uncertainties. The SNAP filter set is designed to observe the same restframe colors for all supernovae from a redshift of 0.1 to 1.7 .

For a mission like SNAP the NIR detectors are critical for achieving the science goals. SNAP has a large visible and NIR focal plane, with 36 CCD detectors and 36 hybridized HgCdTe NIR focal plane arrays (FPAs). The $\mathrm{HgCdTe}$ band-gap is tunable by varying the ratio of $\mathrm{Hg}$ and $\mathrm{Cd}$. The SNAP HgCdTe detectors cutoff at $1.7 \mu \mathrm{m}$, which limits the dark current and sensitivity to thermal radiation from the telescope optics. For a focal plane temperature of $140 \mathrm{~K}$ and uncooled $(290 \mathrm{~K})$ telescope optics, the total dark current plus thermal radiation below $1.7 \mu \mathrm{m}$ is much less than the zodiacal background. The focal plane temperature can be achieved by a passive cooling system, which greatly reduces the complexity and overall mission risk. The HgCdTe diode is bump-bonded to a CMOS multiplexer, where each pixel has its own readout transistor. This is very different from a CCD where all the pixels are read through a single transistor. The hybrid design allows non-destructive 
readout, and multiple sampling modes such as Fowler-N sampling* to reduce the noise. The default readout mode for the SNAP NIR detectors is Fowler-16 sampling. Two vendors are developing $1.7 \mu \mathrm{m} \mathrm{HgCdTe} \mathrm{for}$ SNAP, Rockwell Scientific and Raytheon Vision Systems. The status of the NIR detector development for SNAP is discussed in detail in Schubnell (2006). ${ }^{5}$

Simulations of type Ia supernovae observations for different combinations of NIR detector read noise, dark current, and quantum efficiency are used to constrain the detector performance to meet the science requirements. The requirements are driven by the need to accurately measure the highest redshift supernovae. The simulations presented consider supernovae at a redshift of 1.7, the cutoff for the SNAP supernova program. SNAP will measure supernovae beyond $z=1.7$, but with limited spectrographic information. The simulations show that increased quantum efficiency provides the largest gains in accuracy for supernovae measurements. The impact of improved quantum efficiency is magnified for variable objects like supernovae, which are observed a number of times during the course of their lightcurves. The brightest points are often photon noise dominated and are weighted most heavily in lightcurve fits. $\mathrm{HgCdTe}$ detectors have now achieved internal quantum efficiencies near $100 \%$, and improvements in read noise over the past few years have lead to detectors approaching the SNAP requirements for supernova photometry.

\section{SIMULATION PROCEDURE}

The simulations use a Java based software package called SNAPsim. ${ }^{6}$ The code simulates observations of various astronomical objects with a user defined telescope, instrument, and observing strategy. Optional weather and seeing constraints can be added for ground based observations. For variable objects like type Ia supernovae a template lightcurve is fit to the observations. Multi-color lightcurves are used to derive a distance modulus and dust extinction information for each object. SNAPsim is used to simulate a number of type Ia supernovae at high redshift and study the impact of detector performance on the distance modulus measurements.

The default observing sequence for SNAP is to image each field in all 9 filters on a 4 day cadence. Each observation consists of four, $300 \mathrm{~s}$ dithered exposures for a total integration time of $1200 \mathrm{~s}$. The NIR filters cover twice as much area in the focal plane, and get two such observations at each pointing. The simulation uses aperture photometry, with the aperture size defined by $N_{\text {pix }}$ in Tab. 1.

\subsection{Lightcurve Templates}

SNAPsim lightcurves are simulated using restframe templates for a type Ia supernova in units of $\mathrm{ergs} / \mathrm{cm}^{2} / \mathrm{s} / \AA$ as a function of epoch (from -20 to +70 days from peak) and wavelength. ${ }^{7}$ For epochs greater than 70 restframe days the template is extrapolated using a wavelength dependent decay time that varies from $27-65$ days. The template spectra at peak brightness are shown in Fig. 1 in both the supernova restframe and at a redshift of 1.7.

The templates are normalized to the Vega magnitude system, where the magnitude of Vega at 10 parsecs is 0. The type Ia absolute magnitude is -19.46 for a Hubble constant, $H_{0}=60 \mathrm{~km} \mathrm{~s}^{-1} \mathrm{Mpc}^{-1}$. The Hubble constant sets the overall scale and does not affect any of the results presented below. The absolute source luminosity for each point $(e p o c h, \lambda)$ in the template is

$$
L(\lambda)=F_{s r c}(\lambda) 4 \pi(10 p c)^{2}\left(\frac{\mathrm{h}_{0}}{0.6}\right)^{2} 10^{-(-19.46 / 2.5)} \lambda d(\ln \lambda)
$$

in erg $/ s / \ln (\lambda) . \quad F_{\text {src }}$ is the normalized flux from the templates. The templates assume $\mathrm{h}_{0}=0.6,\left(H_{0} \equiv\right.$ $\mathrm{h}_{0} 100 \mathrm{~km} \mathrm{~s}^{-1} \mathrm{Mpc}^{-1}$ ); the factor of $\mathrm{h}_{0} / 0.6$ corrects the distances for the 'true' value of $\mathrm{h}_{0}$ in the simulation. The final factor of $\lambda d(\ln \lambda)$ converts from per $\lambda$ to per $\ln \lambda$ which preserves the integrated flux when redshifting the source without having to re-normalize. In these units the template is redshifted by simple $z$ scaling $\lambda=$ $\lambda_{\text {restframe }}(1+z)$. The effect of time dilation and stretch is to rescale the time so that $t=t_{\text {template }}(1+z) s$.

After generating the spectra, propagation effects including host galaxy dust, Milky Way dust, atmospheric transmission (for ground based observatories), telescope transmission, filter transmission and detector quantum

\footnotetext{
${ }^{*}$ A Fowler- $\mathrm{N}$ sample is a reset, $\mathrm{N}$ reads, exposure, $\mathrm{N}$ reads. The first and last $\mathrm{N}$ reads are averaged and subtracted to reduce the noise by $\sqrt{N}$ in the absence of $1 / f$ noise.
} 

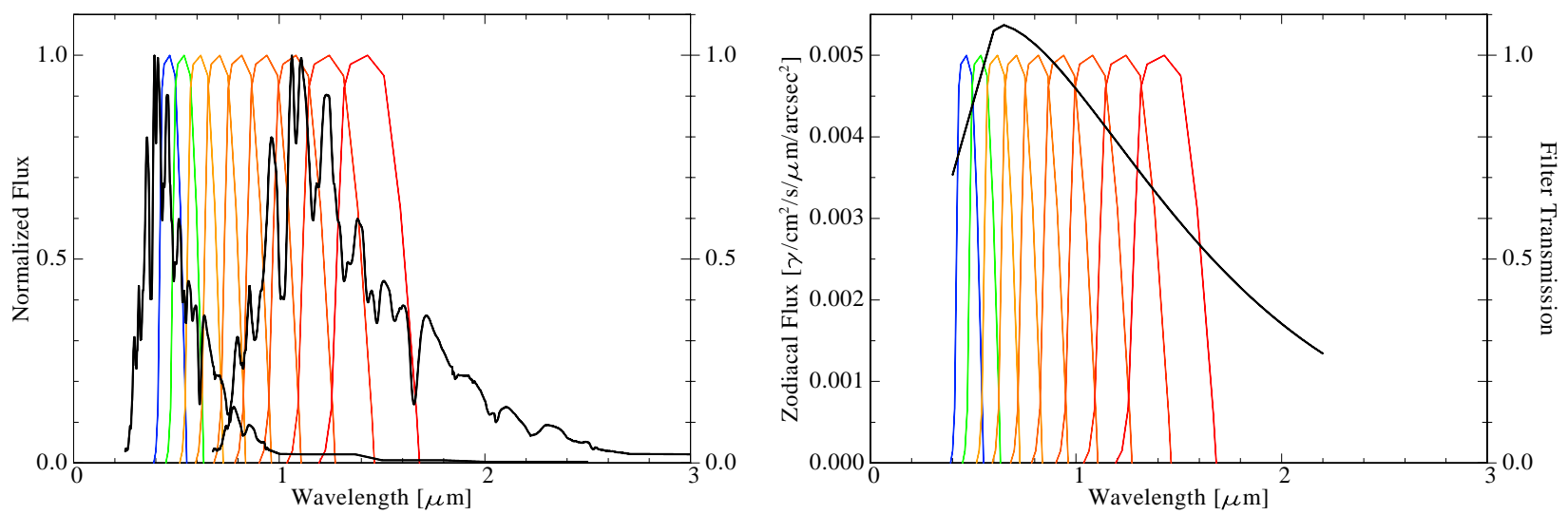

Figure 1. Left panel: Simulated spectrum of a type Ia supernova at peak brightness in the restframe and at $\mathrm{z}=1.7$. Right panel: Zodiacal flux at the north ecliptic pole. Both plots include the 9 SNAP filter bandpasses.

efficiency are applied to the redshifted spectrum. The final step is to calculate the source distance based on the input cosmology. All of the results below assume a flat, $\Lambda \mathrm{CDM}$ cosmology with $\Omega_{\Lambda}=0.7, \Omega_{m}=0.3$, and $w=-1$. The purpose of the simulations is to compare uncertainties on distance modulus to the intrinsic supernova dispersion and the systematic photometric error limit, thus the assumed cosmology does not greatly impact the results. The distance modulus depends on the co-moving distance to each supernovae. For the assumed flat $\Lambda \mathrm{CDM}$ cosmology the co-moving distance is

$$
r(z)=\frac{\mathrm{c}}{\mathrm{H}_{0}} \int_{0}^{z} \frac{d z}{\sqrt{\Omega_{M}(1+z)^{3}+1-\Omega_{M}}} .
$$

The observed flux for a source with luminosity $L$ is

$$
F_{o b s}=\frac{L}{4 \pi r(z)^{2}(1+z)^{2}}
$$

where $r(z)(1+z)$ is the luminosity distance, $d_{L}(z)$. The two factors of $(1+z)$ account for the energy redshift and time dilation. The distance modulus, $\mu$, is proportional to the logarithm of the flux, or in terms of the luminosity distance,

$$
\mu(z) \equiv m-M \propto-2.5 \log _{10} F_{o b s} \propto 5 \log _{10} d_{L}\left(z ; H_{0}, \Omega_{M}, \Omega_{\Lambda}, w_{0}, w^{\prime}\right) .
$$

For the simulations, source flux is measured by the the mean number of photon counts at the detector,

$$
\text { COUNTS }=\mathrm{A}_{\text {tel }} t \int_{\lambda_{\min }}^{\lambda_{\max }} F_{\text {obs }}(\lambda)\left(\frac{\lambda}{\mathrm{hc}}\right) T(\lambda) d \lambda,
$$

where $A_{t e l}$ is the telescope collecting area, $t$ is the exposure time, and $T(\lambda)$ contains all the transmission effects due to dust, atmosphere, filters, optics, and detector quantum efficiency. The factor of $\lambda / h c$ converts from energy units to photons. Calibration information translates the counts into an apparent magnitude in each filter. The variance for each measurement is the mean signal plus sky background and detector noise contributions.

\subsection{Flux Variance and Zodiacal Light}

The redshifted templates and the cosmology from Sec. 2.1 are used to determine the photon counts from the detectors for each epoch through each filter. The flux variance at each epoch is calculated based on the source flux, the background zodiacal light flux, and the detector noise and quantum efficiency. The flux variance is

$$
\sigma^{2}=C O U N T S+F_{z o d i} A_{t e l} A_{P S F} Q E N_{e x p} t+\left(R N^{2}+D C t\right) N_{e x p} N_{p i x},
$$




\begin{tabular}{cccccc}
\hline \hline SNAP Filter & $\begin{array}{c}\text { Central } \lambda \\
{[\mathrm{nm}]}\end{array}$ & $\begin{array}{c}\text { Zodiacal Flux } \\
{\left[\frac{\gamma}{\mathrm{sr} s}\right]}\end{array}$ & $\begin{array}{c}\text { PSF Area } \\
{[\mathrm{sr}]}\end{array}$ & $\begin{array}{c}\text { Zodiacal Rate } \\
{\left[\frac{\gamma}{\mathrm{s}}\right]}\end{array}$ & $N_{\text {pix }}$ \\
\hline 0 & 461.5 & $4.326 \times 10^{11}$ & $8.36 \times 10^{-13}$ & 0.362 & 3.62 \\
1 & 530.8 & $5.927 \times 10^{11}$ & $9.16 \times 10^{-13}$ & 0.543 & 3.96 \\
2 & 610.4 & $7.593 \times 10^{11}$ & $1.02 \times 10^{-12}$ & 0.776 & 4.42 \\
3 & 701.9 & $8.853 \times 10^{11}$ & $1.16 \times 10^{-12}$ & 1.027 & 5.02 \\
4 & 807.2 & $9.837 \times 10^{11}$ & $1.35 \times 10^{-12}$ & 1.328 & 5.84 \\
5 & 928.3 & $8.306 \times 10^{11}$ & $1.59 \times 10^{-12}$ & 1.320 & 6.88 \\
\hline 6 & 1067.5 & $1.112 \times 10^{12}$ & $2.25 \times 10^{-12}$ & 2.502 & 3.31 \\
7 & 1227.7 & $1.118 \times 10^{12}$ & $2.68 \times 10^{-12}$ & 2.996 & 3.95 \\
8 & 1411.8 & $1.080 \times 10^{12}$ & $3.25 \times 10^{-12}$ & 3.506 & 4.78 \\
\hline
\end{tabular}

Table 1. Zodiacal flux at the detector surface (equivalent to $100 \%$ detector QE). The zodiacal rate is per aperture for a point source through each SNAP filter.

where $F_{z o d i}$ is the zodiacal flux in $\gamma / \mathrm{m}^{2} / \operatorname{arcsec}^{2} / \mathrm{s}$ and $A_{P S F}$ is the area of the point spread function (PSF). The PSF is diffraction limited in the NIR, so $A_{P S F}$ grows as $\lambda^{2}$ and the integrated zodiacal light increases for the redder filters despite a declining zodiacal spectrum beyond $0.61 \mu \mathrm{m}$. The exposure time, $\mathrm{t}$, is $300 \mathrm{~s}$ for the SNAP supernova survey, with $N_{\text {exp }}=4$ exposures per pointing. The larger NIR filters get an additional set of 4 exposures at each pointing. Equation (6) assumes the dark current is Poisson distributed and that the detector's conversion gain $\left(\mathrm{e}^{-} / \mathrm{COUNT}\right)$ is one.

The dominant noise source should be the shot noise on the source flux and zodiacal background light, not the detector read noise and dark current contributions. The zodiacal contribution, shown in Fig. 1, is due to sunlight scattered off dust grains. SNAP chooses to survey near the north and south ecliptic poles where the zodiacal background is minimized. Aldering $(2001)^{8}$ studied the observed background in the proposed SNAP north field and found a reddened solar spectrum approximated by a $5800 \mathrm{~K}$ blackbody multiplied by $\lambda^{0.36}$ was a good fit to available data in the visible, but is too faint between 1.25 and $2.2 \mu \mathrm{m}$. A broken log-linear relation tracks the data better beyond $0.6 \mu \mathrm{m}$ where the zodiacal background becomes more important for observing high $z$ supernovae. The best fit to the zodiacal spectrum is

$$
\begin{array}{ll}
f(\lambda)=10^{-17.755} & \text { for } 0.40<\lambda<0.61, \\
f(\lambda)=10^{-17.755-0.730(\lambda-0.61 \mu m)} & \text { for } 0.61<\lambda<2.20,
\end{array}
$$

where $f$ is in units of $\operatorname{erg~cm}^{-2} \mathrm{~s}^{-1} \AA^{-1} \operatorname{arcsec}^{-2}$ This is the zodiacal spectrum used in SNAPsim. The spectrum is convolved with the telescope transmission and the filter response functions (see Fig. 1) then integrated over the filter bandpass to calculate the photon flux at the detector surface, shown in Tab. 1. The filters are logarithmically spaced with $\lambda=\lambda_{0} * 1.15^{n}$ for the $\mathrm{n}^{\text {th }}$ SNAP filter. The CCD pixels are $10.5 \mu \mathrm{m}$ square and the HgCdTe pixels have an $18 \mu \mathrm{m}$ pitch. For a focal length of $21.84 \mathrm{~m}$ this gives a pixel area of $2.31 \times 10^{-13} \mathrm{sr}$ in the visible and $6.79 \times 10^{-13} \mathrm{sr}$ in the infrared, or a plate scale of 0.1 arcsec and 0.17 arcsec, respectively. The PSF area assumes a diffraction limited spot with radius $\theta_{r}=1.22 \lambda / D$ plus charge diffusion, aberrations, and attitude control jitter. The number of pixels for the aperture calculation is given in the last column of Tab. 1. The zodiacal light is an irreducible background that limits the photometric precision and establishes a scale for detector noise; it should be less than the zodiacal background for a 300 s exposure.

\section{DETECTOR DEVELOPMENT AND SIMULATIONS}

The simulations follow the NIR detector development process to explore the effects of performance improvements. The parameters for the NIR detectors are quantum efficiency $(\mathrm{QE})$, read noise $(\mathrm{RN})$ and dark current (DC). Early specifications for the NIR detectors were: $\mathrm{QE} \sim 60 \%$ from $0.9-1.7 \mu \mathrm{m}, \mathrm{RN}=6 e^{-}$, and $\mathrm{DC}<0.1 e^{-} / \mathrm{pix} / \mathrm{s}$. The QE was representative of the best $\mathrm{QE}$ achieved in $1.7 \mu \mathrm{m} \mathrm{HgCdTe}$ (with reasonable noise performance) in 
2001, when detector development for SNAP began. The dark current and read noise specifications ensure that the total detector noise is about half the noise due to the zodiacal background. The read noise was an optimistic goal. The best noise achieved in the first lot of SNAP detectors from both vendors was $12-15 e^{-}$with Fowler-16 sampling. The dark current is limited by cooling the detectors to $140 \mathrm{~K}$. These detector performance specifications are the starting point for the simulation studies.

\subsection{Simulated Lightcurves}

Simulated lightcurves for a $z=1.7$ supernova and the default SNAP detector parameters $(\mathrm{QE} \sim 60 \%, \mathrm{RN}=$ $6 e^{-}$, and $\left.\mathrm{DC}=0.1 e^{-} / \mathrm{pix} / \mathrm{s}\right)$ are shown in Fig. 2. Figure 2 illustrates the importance of NIR observations for high redshift supernovae. The final 3 filters capture most of the photons from this distant source. There is little or no restframe ultra-violet emission redshifted to the optical.

The simulated lightcurves in Fig. 2 are fit to templates with 3 independent parameters, flux at maximum $\left(F_{\max }\right)$, stretch $(\mathrm{s})$, and time of peak brightness $\left(\mathrm{T}_{0}\right)$. The lightcurve fits are used as input to the distance

SN AP Filter 0

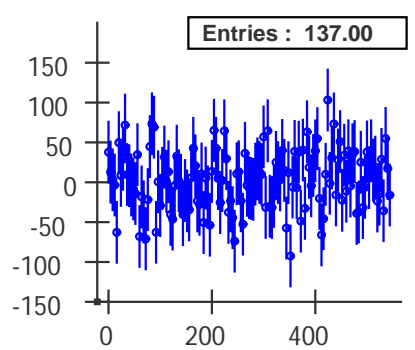

SN AP Filter 1

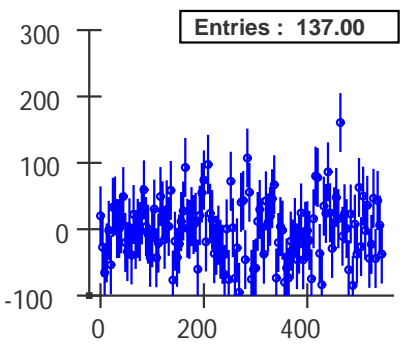

SN AP Filter 2

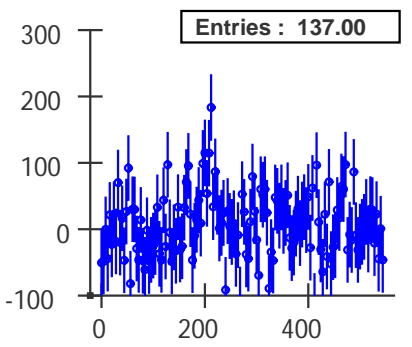

SN AP Filter 3

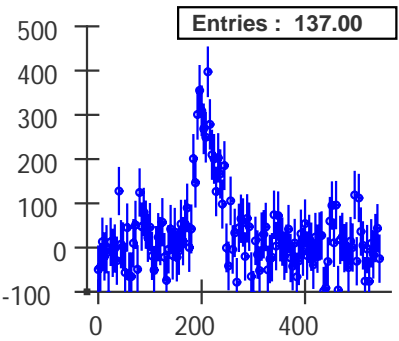

SN AP Filter 4

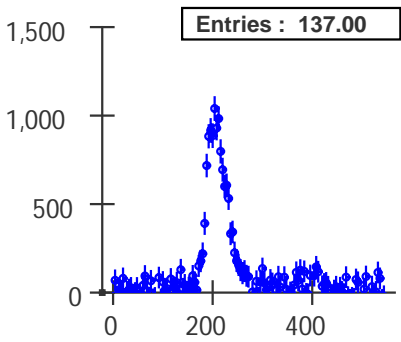

SN AP Filter 5

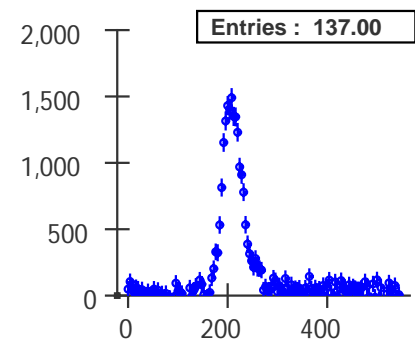

SN AP Filter 6

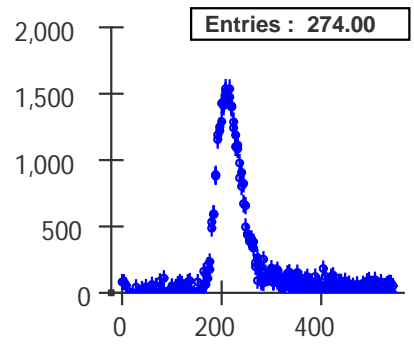

SN AP Filter 7

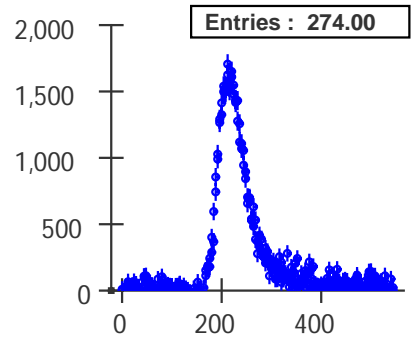

SN AP Filter 8

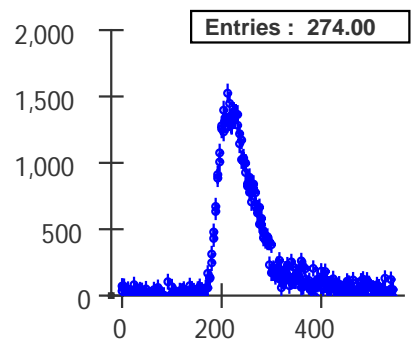

Figure 2. A $z=1.7$ type Ia supernova lightcurve observed every 4 days through the nine SNAP filters. The X axis is in days and the $\mathrm{Y}$ axis is the observed photon counts per $1200 \mathrm{~s}$ observation. 'Entries' specifies the total number of observations for each filter. The larger NIR filters have twice the number of observations, and the NIR detectors are assumed to have $\mathrm{QE}=60 \%, \mathrm{RN}=6 e^{-}$, and $\mathrm{DC}=0.1 e^{-} / \mathrm{pix} / \mathrm{s}$. 

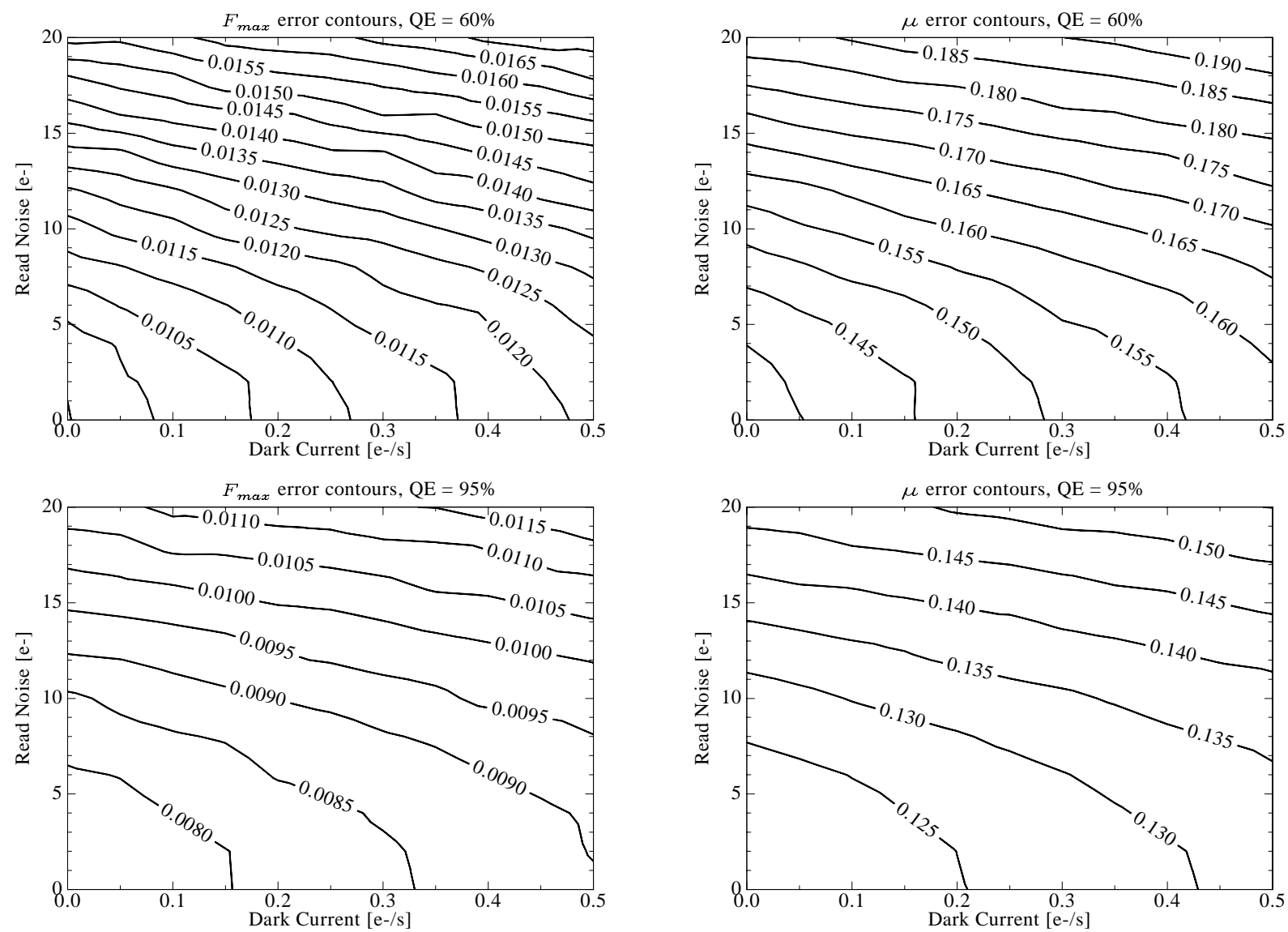

Figure 3. Contours of constant parameter error for the lightcurve parameter $F_{\max }$ (left) and distance modulus, $\mu$, (right) for a $z=1.7$ type Ia supernova. $F_{\max }$ contours are for SNAP filter 8, the reddest SNAP filter. Top plots have QE= 60\%, bottom: $\mathrm{QE}=95 \%$.

modulus $(\mu)$ fitter. The individual lightcurve fits do not account for host galaxy or Milky Way dust extinction, so the dust parameters are fit along with $\mu$. Unlike other groups, SNAP fits the Cardelli, Clayton and Mathis ${ }^{9}$ dust parameters $\mathrm{A}_{V}$ and $\mathrm{R}_{V}$ directly from the multicolor lightcurves, which make the target uncertainties more difficult to achieve. The supernova stretch is also refit using the multi-color information that is now available. Stretch and dust are the two parameters that can introduce the largest errors on $\mu$. Other parameters have a lesser effect and are not considered at this time.

\subsection{Detector Noise Simulations}

The lightcurve fits and distance modulus uncertainty depend on the NIR detector parameters. The effects of RN and DC are simulated for two different detector quantum efficiencies: $60 \%$ and $95 \%$. The simulations in Sec. 3.4 address the effect of quantum efficiency in more detail. 60\% is the best QE achieved at the start of the SNAP $\mathrm{HgCdTe}$ development and $95 \%$ is the maximum QE for $\mathrm{HgCdTe}$ detectors that have an anti-reflective coating. Without an anti-reflective coating the high index of refraction of the CdTe substrate reflects about $20 \%$ of the light; the coating reduces the reflection to $5 \%$ and improves the overall QE. The QE is assumed constant across the entire detector bandpass from $0.9-1.7 \mu \mathrm{m}$. For a fixed QE, contours of constant error for the lightcurve parameters should follow contours of constant total noise, as shown in the left panel of Fig. 3.

Contours of fixed error on $\mathrm{F}_{\max }$ and $\mu$ are plotted as a function of read noise and dark current in Fig. 3, for a type Ia supernova at a redshift of 1.7 . The $F_{\max }$ results are a percent error, and are shown for filter 8 , the 

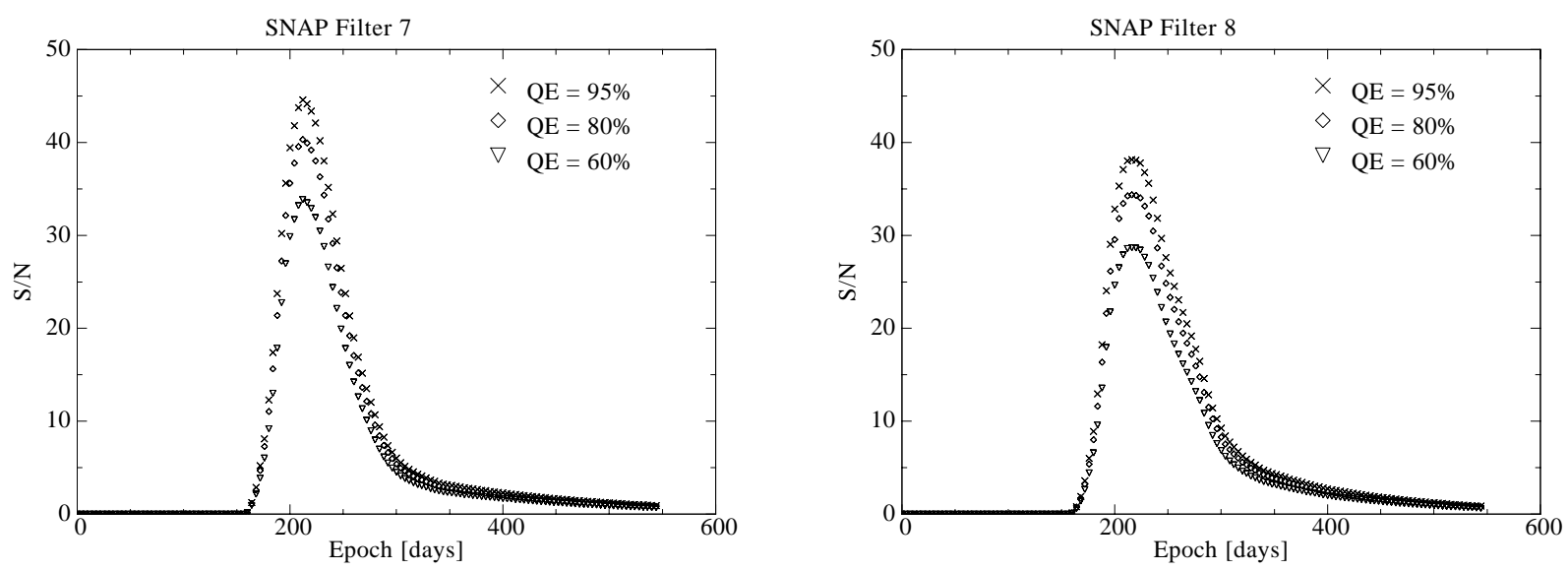

Figure 4. Signal to Noise vs. epoch in SNAP filters 7 (left) and 8 (right), the reddest SNAP filters. The plots assume a total detector noise of $10 e^{-}$per $300 \mathrm{~s}$ exposure. The detection threshold for type Ia supernovae is $\mathrm{S} / \mathrm{N}>3$.

reddest SNAP filter with the lowest $\mathrm{S} / \mathrm{N}$ of the IR filters. The distance modulus is proportional to the logarithm of the peak flux; for small $\sigma_{\mu}$, the percent error is approximately $\sigma_{\mu}$. All calculations of the $\mu$ error use the same visible lightcurves, which are only realized once to save time and ensure that only variations due to the NIR detector performance are studied. For stability, the fit errors are calculated from the average of 4 realizations of each NIR lightcurve.

Figure 3 clearly shows that read noise dominates the detector noise contribution for the parameter space studied. The relatively short $300 \mathrm{~s}$ exposure time lessens the impact of the dark current relative to the $\mathrm{RN}^{2}$ term in Eq. (6). For example, assuming $\mathrm{RN}=8 e^{-}$and $\mathrm{DC}=0.1 e^{-} / \mathrm{pix} / \mathrm{s}$ the total variance due to detector noise for a 4 pixel aperture and four $300 s$ exposures in filter 8 is $1504 e^{-}$. If three $400 s$ exposures are used the variance drops to $1248 e^{-}$, while two $600 \mathrm{~s}$ exposures lowers it to $992 e^{-}$. Fewer, longer exposures are obviously favored for detectors that are read noise dominated. However, the shorter exposure time allows more dithers for better reconstruction of diffraction limited seeing and better cosmic ray rejection, particularly for the visible CCD detectors which do not offer non-destructive readout. The information lost due to cosmic rays and fewer dithers may outweigh the gains from improved detector noise in the NIR.

The importance of high quantum efficiency is also illustrated in Fig. 3. If the QE is only $60 \%$ the total detector noise must be kept below $5 e^{-}$for each $300 \mathrm{~s}$ exposure to achieve a $\mu$ error of 0.14 mag, while the same accuracy can be achieved with more than $15 e^{-}$noise and substantial dark current with high QE detectors. For the $\mu$ errors to approach the stretch corrected intrinsic dispersion of $0.12 \mathrm{mag}$ high QE is essential. Only very long exposures with a $\mathrm{QE}=60 \%$ could achieve this goal. Detailed simulations of quantum efficiency and total detector noise are in Sec. 3.4.

\subsection{Signal vs. Total Detector Noise}

The results from Sec. 3.2 show that read noise dominates the detector noise budget and that the parameter errors follow contours of constant total detector noise. To study the effects of improved QE it is convenient to define the total detector noise as the noise due to RN and DC in a $300 \mathrm{~s}$ exposure. This method has a number of advantages. It is much faster than simulating the entire RN vs. DC parameter space, and the total noise can easily be measured in the laboratory for a variety of different sampling modes available in these multiplexed FPAs. Also, using a measurement of the total noise eliminates the assumption that dark current is Poisson distributed.

Variations in $\mathrm{QE}$ impact the $\mathrm{S} / \mathrm{N}$ for each observation of a supernova lightcurve. In the case of photon or background (zodiacal) limited seeing the first two terms in Eq. (6) dominate, and the noise scales as $\sqrt{Q E}$. Since the signal scales directly with $Q E, S / N \propto \sqrt{Q E}$ for photon limited observations. At peak brightness the 

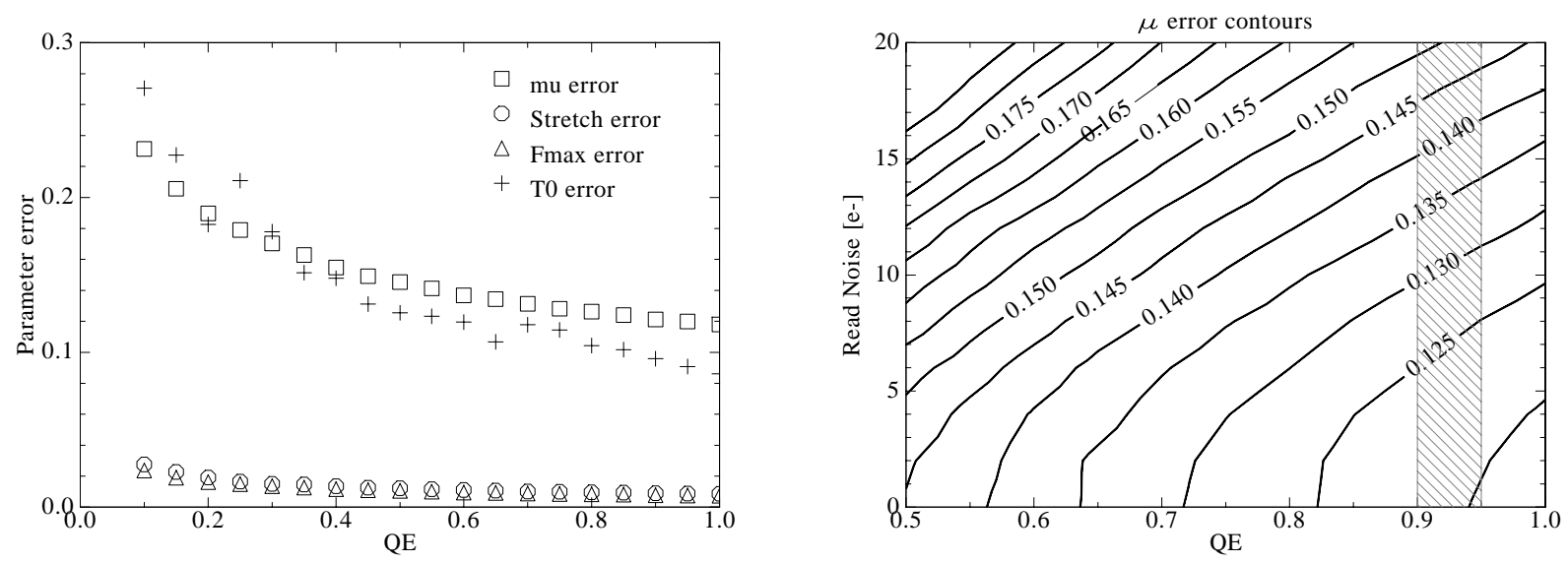

Figure 5. Lightcurve parameter and $\mu$ errors for a $z=1.7$ type Ia supernova assuming noiseless NIR detectors (left). The contours on the right are distance modulus errors for total NIR detector noise up to $20 e^{-}$. The expected QE for SNAP science grade devices is shown in the shaded region.

observations are in this photon dominated regime. $\mathrm{S} / \mathrm{N}$ is simulated by turning off the fluctuations on the signal for each lightcurve point and recording the mean and variance at each epoch. $\mathrm{S} / \mathrm{N}$ for a $z=1.7$ supernova in the last two NIR filters is plotted for selected QEs in Fig. 4. These plots assume a total detector noise of $10 e^{-}$, higher than the initial goal of $6 e^{-}$, but typical of values measured in the second lot of SNAP detectors, see Sec. 3.4.

The $\mathrm{S} / \mathrm{N}$ vs. epoch plots in Fig. 4 are useful for determining the time when a supernova can be identified. The detection threshold for early identification of supernovae candidates is a $\mathrm{S} / \mathrm{N}>3$ on the rising edge of the lightcurve. As the QE improves from $60 \%$ to $95 \%$ each supernova is detected a few observations earlier, leaving additional time to schedule spectroscopic followup.

\subsection{Quantum Efficiency Simulations}

The previous simulations focused on regions of the detector parameter space that represented the performance measured in the first lot of SNAP detectors. The second lot of SNAP detectors showed great improvements in quantum efficiency and a modest reduction in noise. The first improvement was a device with $80 \% \mathrm{QE}$ from $0.9-1.7 \mu \mathrm{m}$ and no anti-reflective coating. With no coating the reflection at the detector surface is $20 \%$; the internal QE of this device was near 100\%. This FPA also had a low single read noise, but high dark current at $140 \mathrm{~K}$ prevented effective noise reduction with Fowler sampling techniques. A second FPA from another vendor also showed high $\mathrm{QE}$ and improved noise. This detector had an anti-reflective coating applied, and the $\mathrm{QE}$ was over $90 \%$ from $0.9-1.7 \mu \mathrm{m}$. The single read noise was slightly higher than the first FPA tested, but this device had very low dark current, and Fowler- 16 sampling at $140 \mathrm{~K}$ reduced the total noise to less than $10 e^{-}$in a $300 \mathrm{~s}$ exposure.

The quantum efficiency simulations explore the QE vs. total detector noise phase space. The simulations are similar to the RN vs. DC simulations above, again assuming a type Ia supernova at $z=1.7$. The simulated data includes a single realization of each lightcurve in the 6 visible filters, followed by 4 realizations of each lightcurve in the NIR filters for each QE and RN pair. The $\mu$ errors combine all the lightcurve fits, and the lightcurve parameter errors are presented for SNAP filter 8, the last NIR filter.

The parameter errors vs. QE are shown in the left panel of Fig. 5 for a noiseless detector. These curves represent the best performance possible for the default SNAP observation strategy of four $300 \mathrm{~s}$ exposures in each filter every four days (eight exposures for the larger NIR filters). The percent error on the lightcurve parameters $F_{\max }$ and $s$ are small and are not greatly impacted by the overall NIR QE. However, the combination of the multiple lightcurve fits produces a distance modulus error that is more sensitive to the NIR QE. The $\mu$ error is the quantity we are most interested in, since it is related to the apparent magnitude observed by SNAP. A 


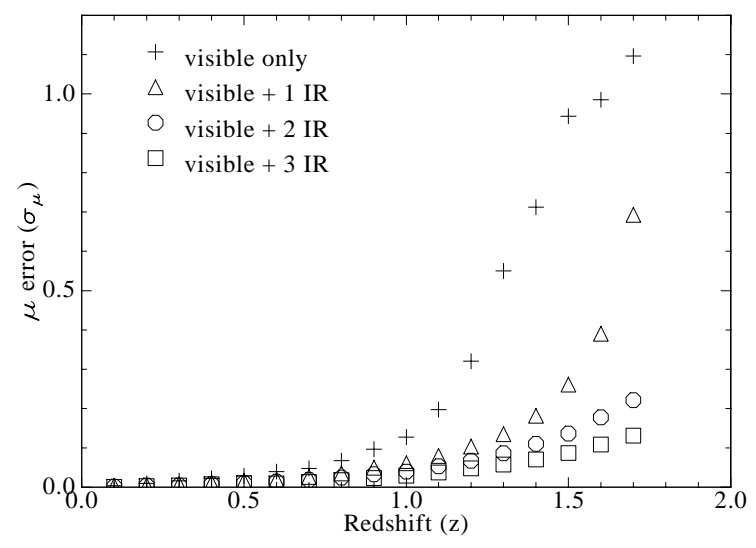

Figure 6. Distance modulus $(\mu)$ errors for type Ia supernovae as a function of redshift and number of filters. Visible denotes the 6 SNAP visible filters, results with 1, 2, or all 3 SNAP NIR filters improve the errors substantially at high $z$. The simulations assume $\mathrm{QE}=90 \%$ and total noise $=10 e^{-}$for the NIR detectors.

more detailed view of the $\mu$ error is shown in the right panel of Fig. 5. These contours in the total noise vs. QE plane show that improved quantum efficiency is the most important factor in achieving precision observations of distant supernovae. By improving the QE from 60 to $80 \%$ the tolerance on the read noise to achieve $\sigma_{\mu}=0.14$ mag increases by more than a factor of two. Reproducing the high quantum efficiency from the second lot of SNAP HgCdTe detectors while striving to reduce the noise is now the goal of the SNAP detector development effort.

\subsection{Importance of NIR}

The data presented thus far shows the ability of a telescope like SNAP to make accurate observations of distant supernovae at a redshift of 1.7. The results shown require a space based instrument with low background in the infrared. For ground based observations the sky background is about 50 times brighter than the zodiacal light level. The results from Fig. 5 cannot be achieved with ground based observations. The effect of excluding the NIR observations is simulated for type Ia supernovae from $z=0.1-1.7$. The NIR observations assume a quantum efficiency of $90 \%$ from $0.9-1.7 \mu \mathrm{m}$ and a total detector noise of $10 e^{-}$, consistent with the best results from the SNAP NIR detector development program. The results for the distance modulus error when excluding one, two, or all three NIR filter observations are plotted in Fig. 6.

The distance modulus error for high redshift supernovae quickly increases in the absence of NIR observations. Excluding just the last NIR filter increases the $\mu$ error by a factor of two at $z=1.7$. Binning 50 type Ia supernovae at $z=1.7$ with a magnitude uncertainty of $\sigma_{\mu}=0.14$ reduces the statistical errors to the $2 \%$ level, the anticipated systematic uncertainty limit. Doubling the $\mu$ error increases the number of supernovae per bin by a factor of four; the long spectroscopic exposure times at high redshift make observing such a large number of supernovae impractical. The decreased photometric accuracy can also lead to larger systematic errors. The NIR observations control systematic uncertainties by observing the same restframe supernovae colors at both high and low redshift. Without the NIR observations the restframe ultra-violet for high redshift supernovae must be calibrated against optical measurements at low redshift. When no NIR observations are available the supernovae at redshifts greater than 1.5 are nearly unusable.

Even for the lower redshift supernovae $(z<1)$ the lack of NIR observations impacts the overall distance modulus error. At low redshifts, observations of the restframe NIR from the supernovae are an excellent indicator of the dust extinction. The scattering due to galactic dust is a strong function of wavelength, leading to large dust extinction in the visible but little in the NIR. The NIR observations for low $z$ supernovae improve the overall $\mu$ error by accurately measuring the dust extinction. The statistical errors at low redshift are small, so 
controlling the systematic uncertainties is even more important. The NIR observations reduce the systematic uncertainties for both the high and low redshift type Ia supernovae.

\section{CONCLUSIONS}

A primary SNAP science goal is to measure the nature of dark energy using type Ia supernovae as standard distance indicators. The high redshift supernovae provide the most leverage on the dark energy parameters, and require accurate NIR measurements. The performance of the SNAP HgCdTe detectors is the most important factor for accurate photometry of high redshift supernovae. The SNAP simulation team has developed a software package capable of quickly simulating a large number of supernovae with a variety of detector and observing parameters. Simulations of high redshift supernovae with the default SNAP observing sequence and varying NIR detector noise and quantum efficiency are used to derive science driven detector performance requirements.

Since the start of the SNAP NIR detector development in 2001 large improvements in detector performance have been realized. Initially much of the development effort was focused on reducing the read noise. However, the second lot of HgCdTe detectors produced for SNAP had high quantum efficiency from two different vendors, Rockwell Scientific and Raytheon Vision Systems. Simulations of the improved quantum efficiency show that the gains in photometric accuracy for type Ia supernovae are much greater when improving quantum efficiency than reducing the detector noise. The SNAP NIR detector specifications now require high quantum efficiency $(>90 \%$ from $0.9-1.7 \mu \mathrm{m}$ ), and continuing development shows promise for reducing the noise even further than the $10 e^{-}$ already achieved. Reducing the noise is more important for spectroscopic observations and measurements of faint objects as part of the SNAP auxiliary science program. For supernovae, the quantum efficiency provides the largest gains because the peak of the lightcurve is photon noise limited. For spectroscopic observations and faint source photometry the zodiacal background and detector noise are the major contributors to the total noise.

The simulations also explore the overall impact of the NIR observations in the redshift range from $0.1-1.7$. SNAP has 9 filter bandpasses, 6 in the visible and 3 in the NIR. Excluding just one of the 3 NIR filters increases the distance modulus error by a factor of two. Without the NIR the high $z$ supernovae needed to discriminate between dark energy models cannot be observed. The NIR is also essential for control of systematic uncertainties. The NIR observations measure dust extinction in low redshift events and provide uniform observations of the restframe B and V band supernovae lightcurves at high redshift. Ground based NIR observations suffer from an atmospheric background that is 50 times brighter than the zodiacal background in space. A dedicated optical and NIR space based instrument like SNAP is the only way to achieve the accuracy required to explore dark energy.

\section{ACKNOWLEDGMENTS}

This work was supported by DOE grant No. DE-FG02-95ER40899. Special thanks to the SNAP simulation team.

\section{REFERENCES}

1. S. Perlmutter, G. Aldering, G. Goldhaber, R. A. Knop, P. Nugent, P. G. Castro, S. Deustua, S. Fabbro, A. Goobar, D. E. Groom, I. M. Hook, A. G. Kim, M. Y. Kim, J. C. Lee, N. J. Nunes, R. Pain, C. R. Pennypacker, R. Quimby, C. Lidman, R. S. Ellis, M. Irwin, R. G. McMahon, P. Ruiz-Lapuente, N. Walton, B. Schaefer, B. J. Boyle, A. V. Filippenko, T. Matheson, A. S. Fruchter, N. Panagia, H. J. M. Newberg, W. J. Couch, and The Supernova Cosmology Project, "Measurements of Omega and Lambda from 42 High-Redshift Supernovae," ApJ 517, pp. 565-586, June 1999.

2. A. G. Riess, A. V. Filippenko, P. Challis, A. Clocchiatti, A. Diercks, P. M. Garnavich, R. L. Gilliland, C. J. Hogan, S. Jha, R. P. Kirshner, B. Leibundgut, M. M. Phillips, D. Reiss, B. P. Schmidt, R. A. Schommer, R. C. Smith, J. Spyromilio, C. Stubbs, N. B. Suntzeff, and J. Tonry, "Observational Evidence from Supernovae for an Accelerating Universe and a Cosmological Constant," AJ 116, pp. 1009-1038, Sept. 1998.

3. G. Aldering et al., "Overview of the SuperNova/Acceleration Probe (SNAP)," in Future Research Direction and Visions for Astronomy. Edited by Dressler, Alan M. Proceedings of the SPIE, Volume 4835, pp. 146-157 (2002)., A. M. Dressler, ed., pp. 146-157, Nov. 2002. 
4. P. Nugent, M. Phillips, E. Baron, D. Branch, and P. Hauschildt, "Evidence for a Spectroscopic Sequence among Type 1a Supernovae," ApJ 455, p. L147, Dec. 1995.

5. M. Schubnell Astronomical Telescopes and Instrumentation, Proc. SPIE, 2006.

6. N. V. Kuznetsova, A. Bonissent, and A. G. Kim, "A Study of SNAP Simulation Lightcurve Fitter Parameters," Bulletin of the American Astronomical Society 207, Dec. 2005.

7. P. Nugent, A. Kim, and S. Perlmutter, "K-Corrections and Extinction Corrections for Type Ia Supernovae," PASP 114, pp. 803-819, Aug. 2002.

8. G. Aldering, "SNAP Sky Background at the North Ecliptic Pole," SNAP Internal Document, 2001.

9. J. A. Cardelli, G. C. Clayton, and J. S. Mathis, "The relationship between infrared, optical, and ultraviolet extinction," ApJ 345, pp. 245-256, Oct. 1989. 